
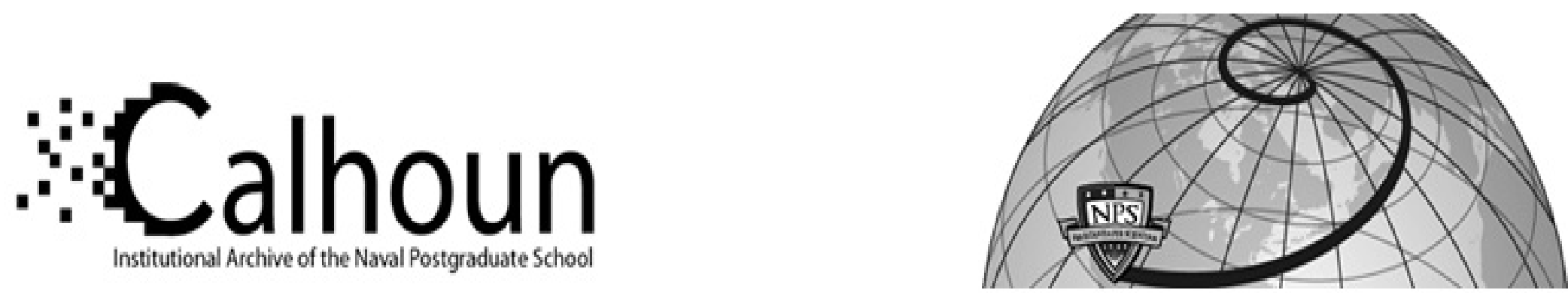

Calhoun: The NPS Institutional Archive DSpace Repository

Sensitivity of tropical cyclone intensification to perturbations in the surface drag coefficient

Thomsen, Gerald; Montgomery, Michael; Smith, Roger

Sensitivity of tropical cyclone intensification to perturbations in the surface drag coefficient, Q. J. R. Meteorol. Soc., in press: 2013, Gerald Thomsen, Michael Montgomery and Roger Smith https://hdl.handle.net/10945/36881

This publication is a work of the U.S. Government as defined in Title 17, United States Code, Section 101. Copyright protection is not available for this work in the United States.

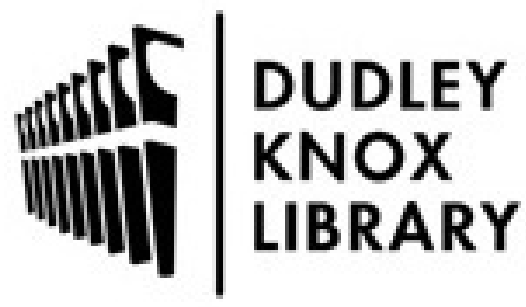

http://www.nps.edu/library
Calhoun is the Naval Postgraduate School's public access digital repository for research materials and institutional publications created by the NPS community. Calhoun is named for Professor of Mathematics Guy K. Calhoun, NPS's first appointed -- and published -- scholarly author.

Dudley Knox Library / Naval Postgraduate School 411 Dyer Road / 1 University Circle Monterey, California USA 93943 


\title{
RMetS
}

Royal Meteorological Society

\section{Sensitivity of tropical-cyclone intensification to perturbations in the surface drag coefficient}

\author{
Gerald L. Thomsen ${ }^{\mathrm{a} \star}$, Michael T. Montgomery ${ }^{\mathrm{b}, \mathrm{c} \dagger}$ and Roger K. Smith ${ }^{\mathrm{a}}$ \\ ${ }^{a}$ Meteorological Institute, University of Munich, Germany \\ ${ }^{\mathrm{b}}$ Department of Meteorology, Naval Postgraduate School, Monterey, California \\ ${ }^{\mathrm{c}}$ Hurricane Research Division, NOAA, Miami, Florida
}

${ }^{\star}$ Correspondence to: G. L. Thomsen, Ludwig-Maximilian’s University, Meteorological Institute, Theresienstrasse 37, Munich 80333, Germany. E-mail: g.thomsen@physik.uni-muenchen.de

${ }^{\dagger}$ The contribution of M. T. Montgomery to this article was prepared as part of his official duties as a US Federal Government employee.

The recent studies of the sensitivity of tropical-cyclone intensification to the surface drag coefficient in a three-dimensional model by Montgomery et al. and Smith et al. are extended to include perturbations of the surface drag coefficient in one of four boundary-layer parametrization schemes: the bulk scheme, the Blackadar scheme, the MRF scheme, and the Gayno-Seaman scheme. The schemes are slightly modified to have the same drag coefficient formulation and the same constant exchange coefficients for sensible heat and moisture. We find that the intensification rate and mature intensity are essentially unaltered when the drag coefficient is perturbed randomly by variations of up to $60 \%$. The results, in conjunction with an analysis of coherent drag coefficient variations for a moving vortex, question the notion that coupled wind-wave models are necessary to accurately forecast tropical-cyclone intensification and mature intensity.

Key Words: hurricanes; typhoons; wind-wave coupling

Received 2 February 2012; Revised 29 August 2012; Accepted 3 September 2012; Published online in Wiley Online Library 11 December 2012

Citation: Thomsen GL, Montgomery MT, Smith RK. 2014. Sensitivity of tropical-cyclone intensification to perturbations in the surface drag coefficient. Q. J. R. Meteorol. Soc. 140: 407-415. DOI:10.1002/qj.2048

\section{Introduction}

The boundary layer of a mature hurricane has been long recognized to be an important feature of the storm as it strongly constrains the radial distribution of vertical motion at its top, as well as those of absolute angular momentum and moisture. Indeed, this idea is central to Emanuel's (1986) formulation of a steady-state hurricane model, to its time-dependent extension (Emanuel, 1997, 2012), and to his theory for the potential intensity of hurricanes (Bister and Emanuel, 1998, and references). More recently, theoretical and observational studies have shown that the maximum tangential wind speed occurs near the top of, but still within, the strong boundary-layer inflow associated with frictional convergence (Braun and Tao, 2000; Zhang et al., 2001; Kepert and Wang, 2001; Montgomery et al., 2006; Kepert, 2006a, 2006b; Smith et al., 2009; Sanger, 2011; Zhang et al., 2012).

Recent work has highlighted the need to further understand the influence of the boundary layer on vortex intensification (Smith and Vogl, 2008; Smith et al., 2009; Smith and Thomsen, 2010; Montgomery et al., 2010; Smith and Montgomery, 2010; Nolan et al., 2010a, 2010b; Smith et al., 2012). In particular, Montgomery et al. (2010) conducted idealized three-dimensional numerical simulations to investigate the sensitivity of tropical-cyclone intensification to changes in the surface drag coefficient in the prototype intensification problem discussed by Nguyen et al. (2008). The study found that, unlike the predictions of previous work using axisymmetric models, the intensification rate of the vortex and the intensity (up to 4 days) increased with increasing $C_{D}$ up to approximately $2 \times 10^{-3}$. When $C_{D}$ was increased 
further, no significant difference in the intensification rate or intensity were found until a threshold of approximately $1.3 \times 10^{-2}$, beyond which the intensity decreased. Although the latter drag coefficient is certainly not realistic over the open ocean, the findings nonetheless suggest the relative insensitivity to the intensification rate and intensity for drag coefficients typical of high wind speeds over the ocean (Powell et al., 2003; Donelan et al., 2004; Black et al., 2007). By relative insensitivity we mean variations that lie within the predictability envelope for intensity associated with the convective structures that operate in and around the eyewall region of the storm (Nguyen et al., 2008).

Of course, it may be objected that the use of a constant drag coefficient is unrealistic and that the results may be prejudiced by the choice of the bulk-aerodynamic boundarylayer scheme in the MM5 model. For this reason, Smith et al. (2012) carried out simulations with a more realistic formulation of the drag coefficient and a range of boundarylayer parametrization schemes and showed that the results found by Montgomery et al. (2010) are robust*. Given the relative insensitivity of the intensification process when $C_{D}$ is doubled in all but the most diffusive scheme (the MRF scheme), we question the viewpoint expressed in Hill and Lackmann (2009, p. 763) that the wave-coupling component in coupled ocean-wave-atmospheric models is necessary to accurately forecast tropical-cyclone intensification and mature intensity.

The current observational estimates of the surface drag coefficient exhibit considerable scatter (Powell et al., 2003; Donelan et al., 2004; Black et al., 2007; Bell et al., 2012). This scatter is presumably some combination of observational error, analysis error and variations in the surface wave field. At this stage it is not possible to determine the relative contribution of these errors, in part because of the different methodologies used to estimate the surface drag coefficient. Extensive efforts have been devoted to including wind-wave coupling for the tropical-cyclone intensification problem (Moon et al., 2004, 2007; Chen et al., 2007; and references therein). However, the results of Montgomery et al. (2010) and Smith et al. (2012) provide a basis for questioning the need for such a complex approach and suggest that perhaps the results of numerical models will not be unduly sensitive to such spatial fluctuations in the drag coefficient.

In this article we investigate the sensitivity of the intensification process to random spatial fluctuations in the drag coefficient and compare the associated variability in the intensity to that caused by moisture perturbations in the boundary layer. The variations in the drag coefficient have small scale, specifically the scale of the grid, and cover the range of amplitudes typically found in laboratory experiments as well as that based on observational uncertainty from different suites of dropwindsonde data. This method is one way to address the sources of variability in the drag coefficient discussed above. To apply the moisture perturbations, we use the simple model configuration presented by Nguyen et al. (2008). In the first suite of experiments there is no background flow.

Of course, the drag coefficient may have more spatially coherent variability associated with vortex-scale asymmetries in the surface wind field and in the

\footnotetext{
*The boundary-layer schemes in that study were modified slightly to have the same drag coefficient formulation and for simplicity the same constant exchange coefficients for sensible heat and moisture.
}

corresponding distribution of surface stress (e.g. Moon et al., 2004). For this reason, we analyse also calculations of the drag coefficient in the idealized tropical-cyclone model with a uniform background flow described by Thomsen et al. (2012). In this situation, the drag coefficient has a large-scale, azimuthal wavenumber-one asymmetry on account of the stronger winds on the right-hand side relative to the storm motion in an earth-relative frame, but it has variability also on account of the intrinsically stochastic component of surface wind heterogeneities associated with rotating deep convection in the model. To examine this issue, two additional sets of calculations are carried out to assess the influence of a coherent spatial pattern of the drag coefficient. These calculations are detailed further below.

In section 2 we describe briefly the numerical model. The calculations and supporting interpretations of them are described in sections 3 and 4 . Our conclusions are given in section 5 .

\section{The numerical experiments}

\subsection{The model}

The numerical experiments are similar to those described in Nguyen et al. (2008). They are carried out using a modified version of the Pennsylvania State University/National Center for Atmospheric Research mesoscale model MM5 (version 3.6.1). A detailed description of the model can be found in Grell et al. (1995). The model is configured with three domains: a coarse mesh of $45 \mathrm{~km}$ grid spacing and two two-way nested domains of 15 and $5 \mathrm{~km}$ grid spacing. The domains are square and are $9000 \mathrm{~km}, 4500 \mathrm{~km}, 1500 \mathrm{~km}$ on each side. The calculations are performed on an $f$-plane centred at $20^{\circ} \mathrm{N}$. In all calculations there are $24 \sigma$-levels in the vertical, 11 of which are below $850 \mathrm{mb}$ (Smith and Thomsen, 2010 , section 2.1 ). The vertical resolution in the boundary layer is believed to be adequate for correctly representing the boundary-layer dynamics under the prescribed changes to the surface exchange coefficients and vertical mixing of heat and momentum. There is no representation of dissipative heating.

Deep moist convection is resolved explicitly and represented by the warm-rain scheme as in Montgomery et al. (2010). In addition, we choose one of four boundarylayer schemes available in the model as detailed in subsection 2.2 (also in the appendix of Smith and Thomsen, 2010, and references therein). The warm-rain and boundarylayer schemes are applied in all domains. No cumulus parametrization is used. The sea surface temperature is a constant $\left(27^{\circ} \mathrm{C}\right)$. We use the simple radiative cooling scheme available in MM5, which imposes a temperature-dependent cooling rate on the order of $1-2{ }^{\circ} \mathrm{C} \mathrm{day}^{-1}$.

\subsection{The boundary-layer and surface-layer schemes}

The four boundary-layer schemes examined here are the bulk scheme, the Blackadar scheme, the Gayno-Seaman scheme and the MRF scheme. To facilitate a proper comparison of the schemes in the four main calculations, the surface drag and heat and moisture exchange coefficients are modified to fit the results of the Coupled Boundary-Layer Air-Sea Transfer experiment (CBLAST: Black et al., 2007; Drennan et al., 2007; French et al., 2007; Zhang et al., 2008). The surface exchange coefficients for sensible heat and moisture 
(a)

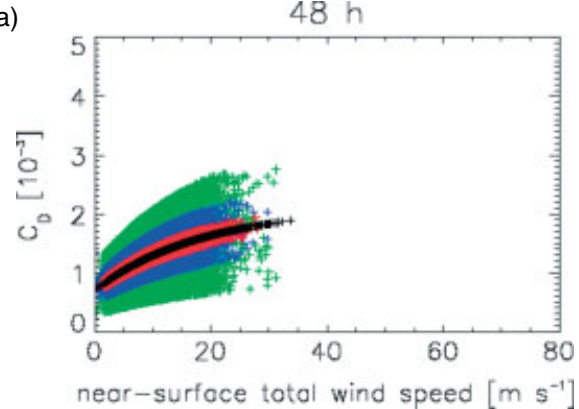

(b)

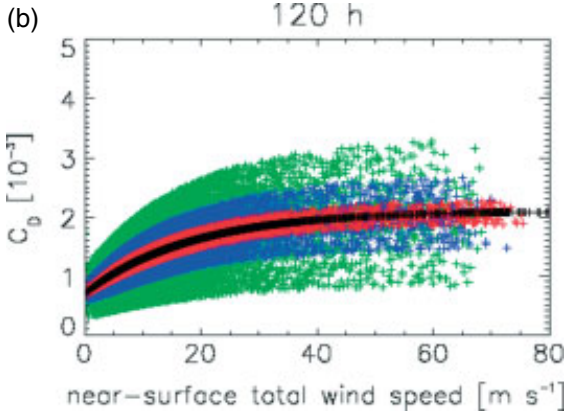

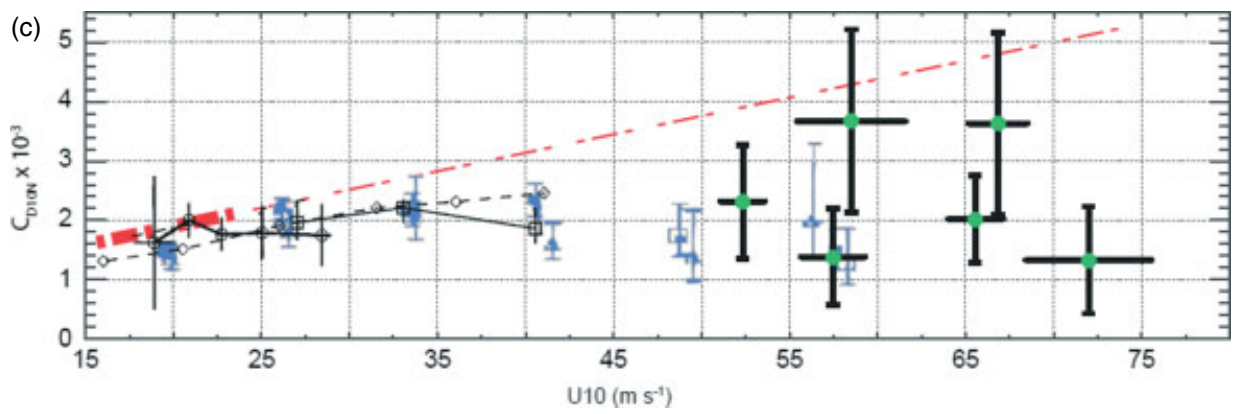

Figure 1. Scatter plots of $C_{D}$ versus total wind speed (a) at $48 \mathrm{~h}$ and (b) at $120 \mathrm{~h}$ in the calculations with the Blackadar scheme for the control run ( $\alpha=0$; black plots), $\alpha=0.1$ (red plots), $\alpha=0.3$ (blue plots), and $\alpha=0.6$ (green plots). (c) shows the wind-speed dependence of $C_{D}$ from the recent study by Bell et al. (2012) (solid black lines; green circles give mean estimates for a particular hurricane mission) along with a comparison with previous studies (courtesy of Bell et al., 2012). Light black symbols are adapted from French et al.(2007) and blue symbols from Vickery et al. (2009). The red line indicates the measured (bold) and extrapolated (thin) Large and Pond (1981) drag coefficient.

are set to the same constant, $1.2 \times 10^{-3}$. For the exchange coefficient of momentum, the drag coefficient is given by the formula:

$$
\begin{array}{r}
C_{D}=\left[0.7 \times 10^{-3}+1.4 \times 10^{-3}\{1-\exp (-0.055|\mathbf{u}|)\}\right] \\
\times(1+\alpha R),
\end{array}
$$

where $|\mathbf{u}|$ is the wind speed at the lowest model level.

A random perturbation is applied through the constant $\alpha$ in Eq. (1), set to $0.1,0.3$ or 0.6 and the random number $R \in[-1,1]$. This random number is set for each grid point individually and new random values are derived every $15 \mathrm{~min}$ throughout the simulation. The drag coefficient and the exchange coefficients for heat and moisture were modified directly in the bulk scheme and were modified indirectly in the Blackadar, MRF and Gayno-Seaman scheme as described in the appendix of Hill and Lackmann (2009). In essence, the friction velocity $U_{*}$ is replaced by $V_{0} \sqrt{C_{D}}$, where $V_{0}$ is the total wind speed at the lowest model level.

\subsection{Variability of the randomly perturbed surface drag coefficient compared to observations}

As a check on whether the scatter in the perturbed drag coefficient is consistent with the observed scatter in the observationally derived drag coefficient, we show in Figure 1 two scatter plots of the surface drag coefficient as a function of surface wind speed at the lowest model level at different times, both for the control experiment and the experiment with perturbations in $C_{D}$ using $\alpha=0.1,0.3,0.6$. As noted above, the small-scale variations applied cover the wide spectrum of amplitudes typically seen in laboratory experiments as well as the observational uncertainty from different suites of dropwindsonde data. The value of $\alpha=0.6$ gives a variability in $C_{D}$ that serves as the upper bound to the observed variability at the present time (Figure 1(c); Bell et al., 2012, give details) and to variability found in recent laboratory experiments (Donelan et al., 2004).

\subsection{Experimental design}

The initial vortex is axisymmetric with a maximum tangential wind speed of $15 \mathrm{~m} \mathrm{~s}^{-1}$ at the surface at a radius of $120 \mathrm{~km}$. The magnitude of the tangential wind decreases sinusoidally with height, vanishing at the top model level. The temperature field is initialised to be in gradient wind balance with the wind field using the method described by Smith (2006). The far-field temperature and humidity are based on Jordan's Caribbean sounding for the hurricane season (Jordan, 1958).

A set of ensemble calculations is carried out for the Blackadar scheme, which, as shown by Smith and Thomsen (2010) appears to have the most realistic values of vertical eddy diffusivity (cf. Zhang et al., 2011). These runs are similar to the main calculations, but have a $\pm 0.5 \mathrm{~g} \mathrm{~kg}^{-1}$ random perturbation added to the water-vapour mixing ratio up to $950 \mathrm{mb}$ at the initial time. In order to keep the mass field unchanged, the temperature is adjusted at each point to keep the virtual temperature unchanged. A fourmember ensemble is carried out for $\alpha=0$ to generate a representative envelope of uncertainty for the experiments.

It is well known that a moving vortex produces a wave response that is a prominent azimuthal wavenumber-one asymmetry, with a corresponding structure in the drag coefficient (e.g. Moon et al., 2004, and references therein). To assess the implications of such an asymmetry, we carry out a series of additional experiments in which there is a uniform flow, similar to the calculations presented in Thomsen et al. (2012), using the four boundary-layer schemes discussed above. In addition, we conduct two 
sets of companion experiments. In the first set, a uniform translation speed is added to the surface wind speed in the calculation of the drag coefficient (Eq. (1)). For this first set, the vortex is approximately stationary, but the drag coefficient has an azimuthal wavenumber-one asymmetry similar to that found in a moving vortex. In the second set, we consider a vortex embedded in a unform flow. The spatial structure of the drag coefficient is then modified by (i) subtracting the uniform flow in the calculation of the drag coefficient (Eq. (1)), thus largely removing the azimuthal wavenumber-one translation asymmetry from the drag coefficient, and (ii) subtracting the uniform easterly winds from the meridional wind field. The latter modification has the effect of shifting the area of maximum drag coefficients to the front right quadrant and the area of minimum drag coefficients to the rear left quadrant. Together, both suites of experiments provide us with a stringent test of the influence of both a coherent spatial pattern of drag and random perturbations thereto. In the moving vortex experiments, the surface fluxes of sensible and latent heat are set to zero in the outer domains and the radiation scheme is formulated slightly differently (section 4 here and Thomsen et al., 2012, give details).

Figure 2 illustrates the spatial distribution in the drag coefficient and the horizontal wind speeds at the lowest model layer in selected experiments. The fields are shown at the first output time (15 min), well before deep convection has commenced. Panel (a) shows the situation in which there are no perturbations to the drag coefficient $(\alpha=0)$. The wind and drag at this time have an axisymmetric structure. In panel (b), the surface drag coefficient is randomly perturbed with $\alpha=0.6$, but the winds at this time are still axisymmetric. Panel (c) shows the situation for a translating vortex in a $5 \mathrm{~m} \mathrm{~s}^{-1}$ easterly flow (from Thomsen et al., 2012). As expected, the fields show a prominent pattern of enhanced winds on the northern side of the vortex and a corresponding enhancement of the drag coefficient in the same region. Panel (d) shows the situation for a moving vortex that has been modified as described for set (ii). The fields show a prominent pattern of enhanced drag coefficients in the front right quadrant. With our experiments in which the maximum drag is located to the right and front right of the vortex motion vector in two different experiments, we cover the situation shown by Moon et al. (2004). The area of maximum drag coefficients is located between the right and front right of the motion vector in their experiments in which the surface drag coefficient was calculated from their wave model below a simulated tropical cyclone.

\section{Results from experiments with random perturbations to the drag coefficient}

Since the azimuthal average of the perturbations in the drag coefficient is nearly zero, some readers would argue it would not be a big surprise if there were only small variations in the maximum azimuthally-averaged tangential and maximum azimuthally-averaged inflow wind field. In fact, plots of these quantities do show such insensitivity. We do not show these plots here, but rather want to concentrate on the maximum local wind speed at any given time. Figure 3 shows a time series of the maximum wind speed in the boundary layer for the four experiments $(\alpha=0.0,0.1,0.3,0.6)$ and with each of the four boundary-layer schemes described above. The salient points to note are as follows.
The maximum wind speed increases steadily with time as the vortex intensifies, following a gestation period lasting approximately $9 \mathrm{~h}$ for all schemes. It is significant that changing the boundary-layer scheme while keeping the surface-layer formulation unchanged has a larger impact on the intensification rate and mature intensity of the vortex than perturbing the drag coefficient. This result is in line with the results of Smith and Thomsen (2010).

Recalling the results of Nguyen et al. (2008), there is variability in the intensification curves associated with the random nature of rotating deep convective structures. Nguyen et al. illustrated the intrinsic variability of the intensification process by carrying out a series of ensemble experiments in which a small-amplitude random perturbation was added to the boundary-layer moisture field in the initial condition. These authors pointed out that, because of this intrinsic variability, one should be cautious about drawing general conclusions from a comparison of just two deterministic calculations. Moreover, if the difference between the intensity curves for two deterministic calculations lies within the envelope of uncertainty of the ensemble, then one would judge that the difference in the outcome is not significant. For this reason we carry out next a modest set of four ensemble calculations that adequately span the range of variability in the simulated intensification process (e.g. Thomsen et al., 2012).

Figure 4 shows the time evolution of the maximum wind speed for the perturbed moisture ensemble calculations using the Gayno-Seaman boundary-layer scheme and for the case of no perturbation in the drag coefficient (i.e. $\alpha=0$ ). The moisture ensemble is generated by the procedure described above. From the figure we infer an envelope of intensity uncertainty of approximately $15 \mathrm{~m} \mathrm{~s}^{-1}$. Since the differences in intensity amongst the experiments with perturbed drag coefficient are comparable to that associated with the moisture ensemble (cf. Figure 4), the differences with randomly perturbed drag coefficient cannot be regarded as significant.

\section{Results from experiments with coherent perturba- tions to the drag coefficient}

The previous experiments modelled the influence of surface waves as purely a random variation in the drag coefficient without a coherent spatial pattern. However, as noted in section 2, a moving vortex produces a response in the surface wave field that exhibits a prominent azimuthal wavenumber-one asymmetry around the storm, with a corresponding coherent structure component in the drag coefficient (e.g. Figures 9 and 12 of Moon et al., 2004).

To address this issue, we show in Figure 5 time series of maximum wind speeds in the first suite of calculations with a coherent pattern of variation in the drag coefficient for a stationary vortex as described in section 2.4. The time series for the control run (black curves) are superimposed for comparison. The difference in the wind speed is typically less than $10 \mathrm{~m} \mathrm{~s}^{-1}$, but no more than $15 \mathrm{~m} \mathrm{~s}^{-1}$.

Figure 6 shows time series plots of maximum winds for the second suite of calculations with a moving vortex. The experiments with a background flow and a removed wavenumber-one asymmetry in the surface drag coefficient (described in section 2.4) are shown by the blue curves. The experiments with the maximum drag located in the front right quadrant are shown by the red curves. These curves 
(a)

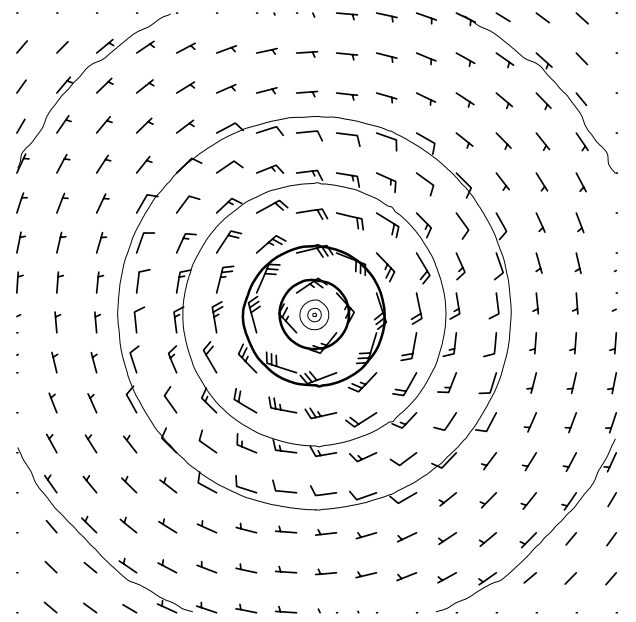

(c)

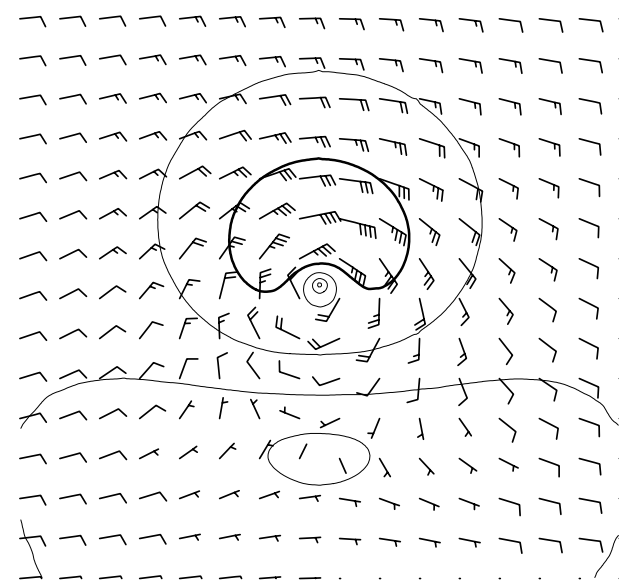

(b)

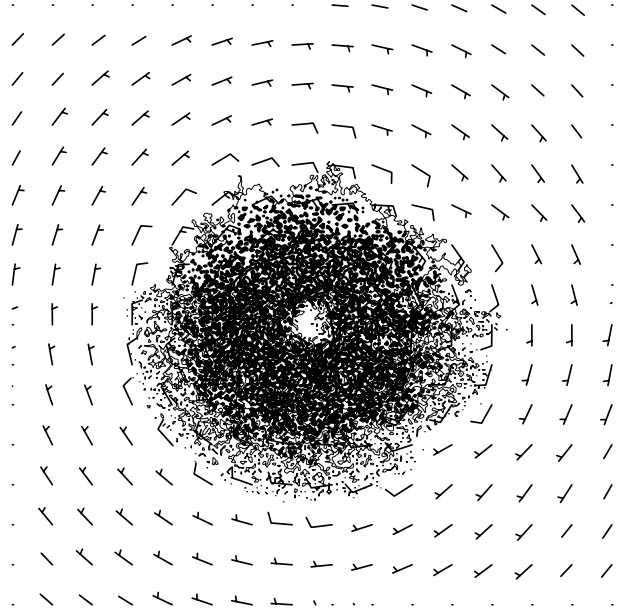

(d)

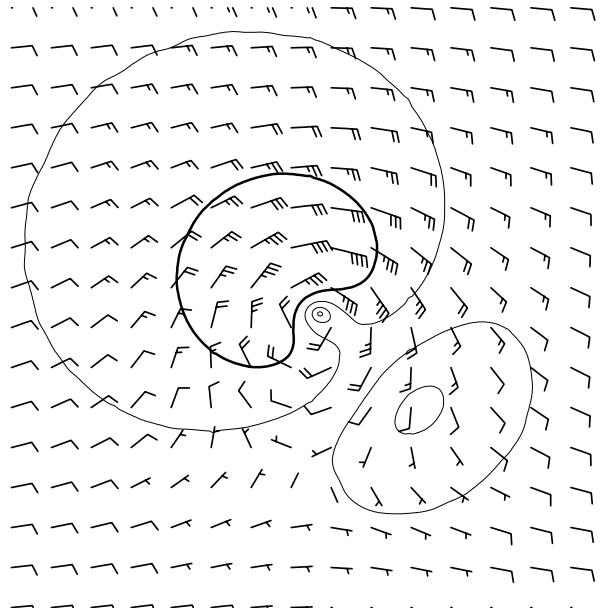

Figure 2. Plot of the surface drag coefficient (with thin contour interval of $2 \times 10^{-4}$ up to $12 \times 10^{-4}$, and bold contours of $14 \times 10^{-4}$; lines below $12 \times 10^{-4}$ are not shown in (b)) and horizontal wind vectors (full barbs $5 \mathrm{~m} \mathrm{~s}^{-1}$ ) at $850 \mathrm{mb}$ at the first output time (after 15 min simulation time) in the inner domain (1500 km squared). (a) Blackadar scheme, $\alpha=0$; (b) Blackadar scheme, $\alpha=0.6$; (c) Blackadar scheme, $\alpha=0$, with uniform background easterly wind field of $5 \mathrm{~m} \mathrm{~s}^{-1}$; and (d) Blackadar scheme, $\alpha=0$, with uniform background easterly wind field of $5 \mathrm{~m} \mathrm{~s}^{-1}$, but with $5 \mathrm{~m} \mathrm{~s}^{-1}$ having been subtracted from the meridional wind field in the calculation of the drag coefficient.

are compared with our previous results for a moving vortex (Thomsen et al., 2012; black curves). The difference in the maximum wind speed is typically less than $10 \mathrm{~m} \mathrm{~s}^{-1}$. Note that the vortices with a background flow are less intense throughout most of the time shown than in the case of a stationary vortex (cf. Figure 5). This finding is at first sight in contradiction to those of Thomsen et al. (2012), but can be explained by the fact that the surface fluxes of sensible and latent heat in the intermediate and outer domains are set to zero in the runs with a moving vortex. The fluxes of sensible and latent heat are turned off in the outer two domains to suppress the tendency of the model to build up large values of conditional available potential energy (CAPE) associated with the ambient zonal flow. Without such a specification, the ambient CAPE would increase with background wind speed and time. In contrast to the radiative cooling scheme used for the experiments described in section 3 and for those with a stationary vortex described in this section, we use a simple Newtonian scheme for the experiments with a moving vortex. In this scheme, the temperature is relaxed back to its initial profile ${ }^{\dagger}$ on a time-scale of one day. This

${ }^{\dagger}$ This profile has to be defined on pressure coordinates. On any other coordinate system, the temperature would be allowed to change independent of pressure, thus inducing an artificial thermal circulation. modification is necessary to avoid inducing an artificial thermal circulation between southern and northern sides of the model domain. The runs without a background wind speed do not have these modifications in this study. For the above reasons, the graphs shown in Figures 5 and 6 are not directly comparable.

In summary, for each suite of experiments, either with or without a background wind field, the evolution of the vortex intensity lies within a comparatively narrow envelope of variability (comparable with that in Figure 3 ). Based on these results, we conclude that the precise spatial structure of $C_{D}$ is not as important as suggested by previous studies.

While there is no apparent change in the rate of vortex intensification or final intensity of the vortex with a structural perturbation to the drag coefficient, it is pertinent to ask if such a modification can impact the predicted track of a storm. Figure 7 shows the track predictions from the experiments with a moving vortex. For the purpose of calculating the track of a moving vortex, the vortex centre is defined as the centroid of relative vorticity at $900 \mathrm{mb}$ over a circular region of $200 \mathrm{~km}$ radius from a 'first-guess' centre, which is determined by the minimum of the total wind speed at $900 \mathrm{mb}$. The experiments with a background flow and a removed wavenumber-one asymmetry in the surface drag coefficient (described in section 2.4) are shown by the blue 

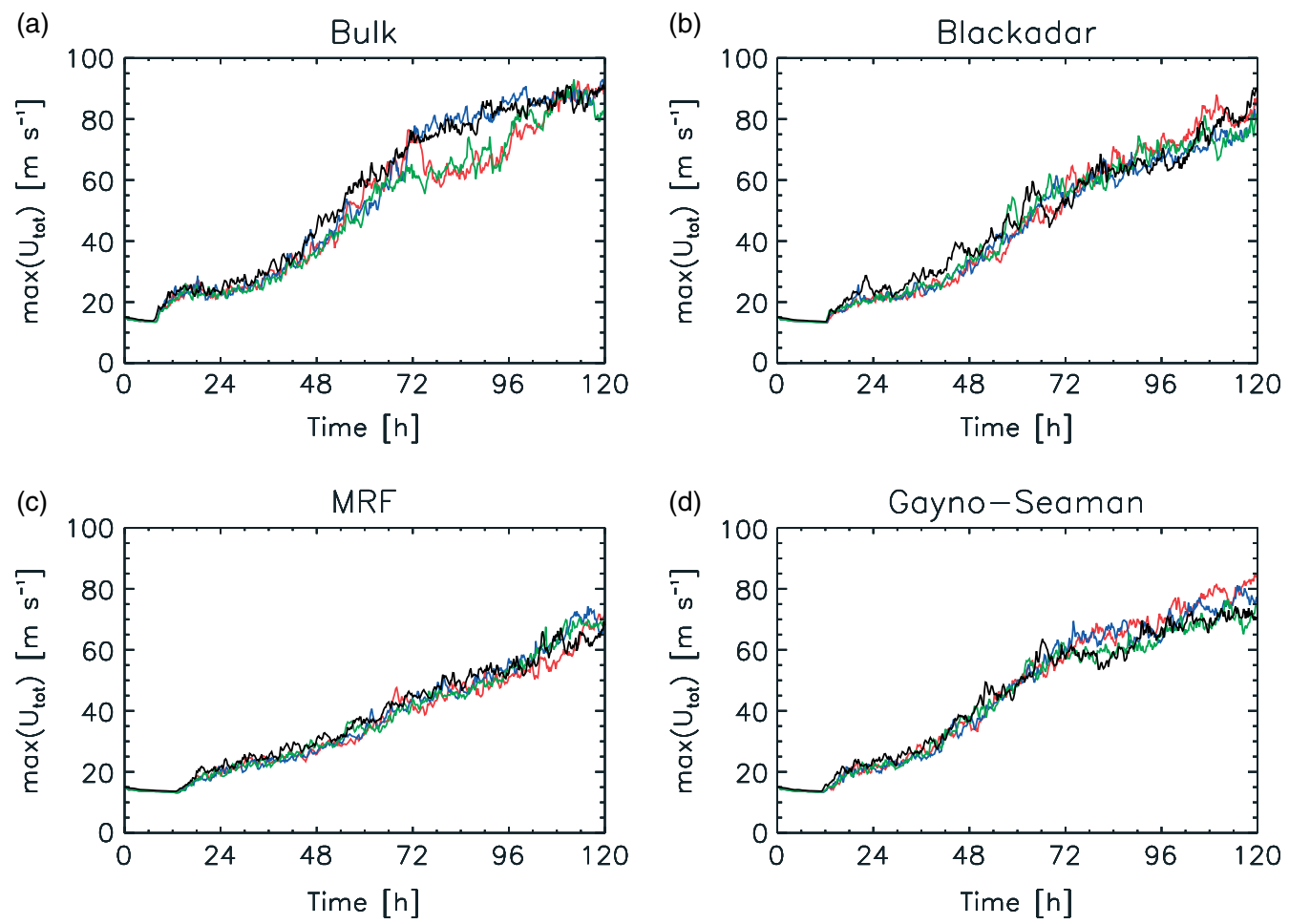

Figure 3. Time series of the maximum wind speed in the stationary vortex calculations with (a) the bulk scheme, (b) the Blackadar scheme, (c) the MRF scheme, and (d) the Gayno-Seaman scheme: the control run ( $\alpha=0$; black curve), $\alpha=0.1$ (red curve), $\alpha=0.3$ (blue curve), and $\alpha=0.6$ (green curve).

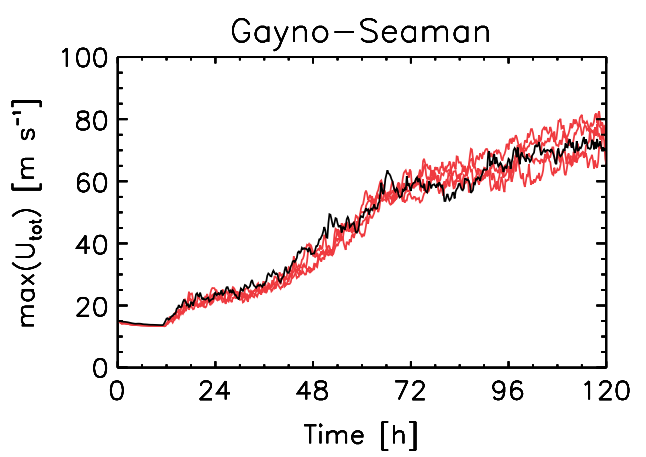

Figure 4. Time series of maximum wind speed in the stationary vortex calculations with the Gayno-Seaman scheme: control run as seen in Figure 3 (black curve), and four ensemble runs with random moisture perturbations (red curves). $\alpha=0$

curves. The experiments with the maximum drag located in the front right quadrant are shown by the red curves. These curves are compared with our previous results for a moving vortex (Thomsen et al., 2012; black curves). The south-north variations in the storm tracks are generally below $50 \mathrm{~km}$ up to approximately $72 \mathrm{~h}$ of the calculation (corresponding roughly to $1500 \mathrm{~km}$ westwards translation). The variations are still below $50 \mathrm{~km}$ until the end of the calculation in the experiments with the bulk and the MRF scheme, while the variation grows up to $100 \mathrm{~km}$ in the calculations with the Blackadar and the Gayno-Seaman scheme. Figure 8 shows the track predictions from a set of ensemble experiments with the bulk scheme. The individual members of this set of four additional calculations have been generated as described in section 2.4. The north-south variation of the predicted storm tracks lies within the envelope of the predicted tracks in Figure 7(a) at all times. We conclude that the choice of boundary-layer parametrization has a larger possible impact on the track prediction of a tropical cyclone than the spatial distribution of the surface drag coefficient.

\section{Conclusions}

We have carried out idealized three-dimensional numerical simulations to investigate the sensitivity of tropical-cyclone intensification to random and structural perturbations in the surface drag coefficient. The study extends those of Montgomery et al. (2010) and Smith et al. (2012), which suggested that the intensification rate and mature intensity in a regime of realistic values of the drag coefficient may not be unduly sensitive to the precise values of the drag coefficient. We presented plausible and compelling evidence that wind-wave models may not be essential for the accurate prediction of tropical-cyclone intensification and tracks.

When the baseline drag coefficient was perturbed randomly by variations of up to 10,30 or even $60 \%$, we found that the intensification rate and mature intensity were well within the envelope of uncertainty of four additional ensemble experiments. Since one cannot use simulations with such random variations alone to question the need for a wave model, we performed two additional suites of experiments. These experiments included coherent modifications of the drag coefficient, such as might arise for a translating storm, and an associated wind-driven wave field at the ocean surface. We obtained similar results from these experiments and the results thereof were found to be broadly independent of the boundary-layer scheme used in the model.

A comparison of the tracks calculated for the experiments with a moving vortex showed negligible sensitivity to the spatial distribution of the surface drag coefficient. 

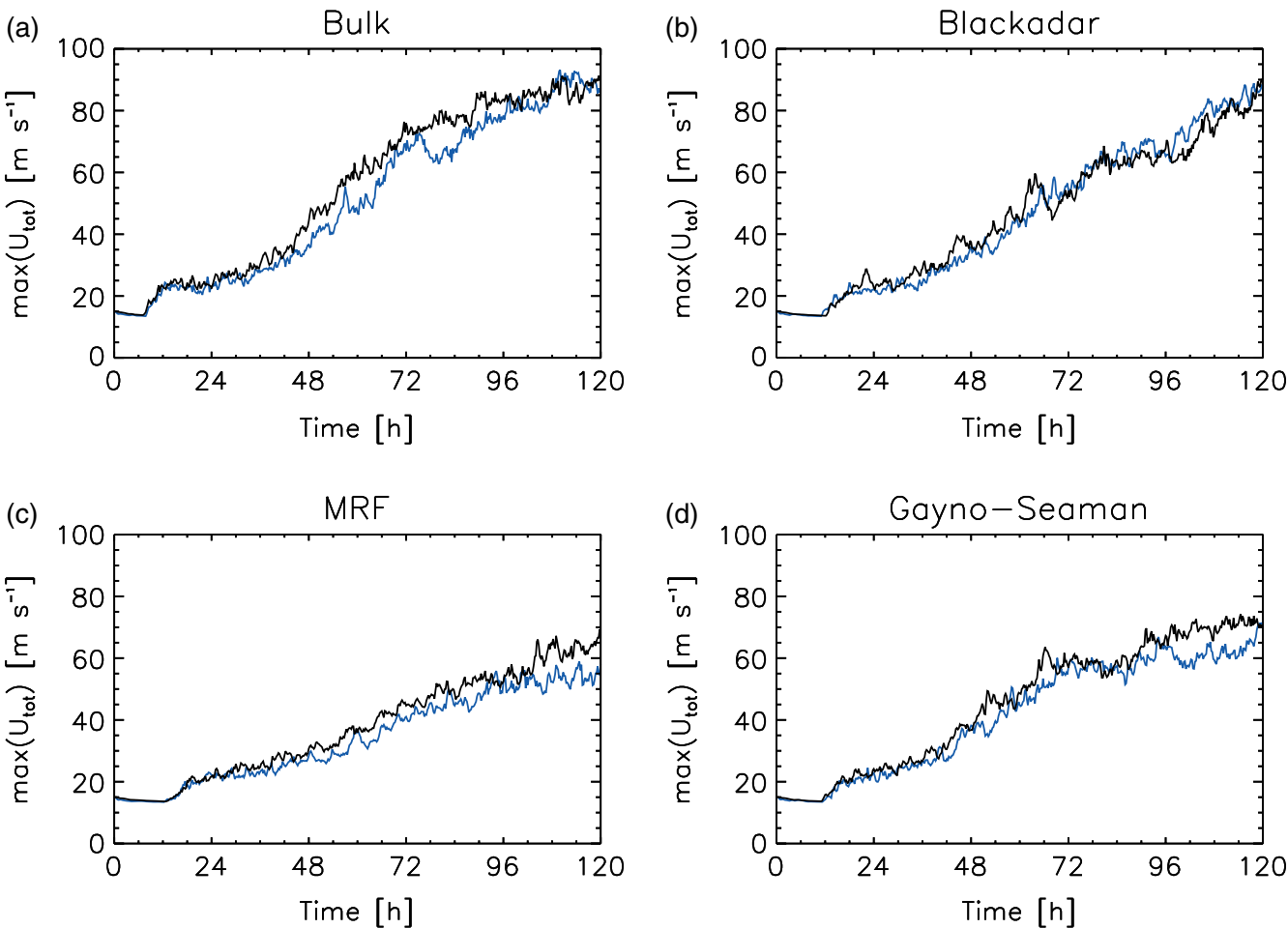

Figure 5. Time-series of maximum wind speed in the calculations for a stationary vortex with (a) the bulk scheme, (b) the Blackadar scheme, (c) the MRF scheme, and (d) the Gayno-Seaman scheme: the control run (black curve), and control run with the zonal wind field reduced by $5 \mathrm{~m} \mathrm{~s}{ }^{-1}$ for the calculation of the surface drag coefficient (blue curve).
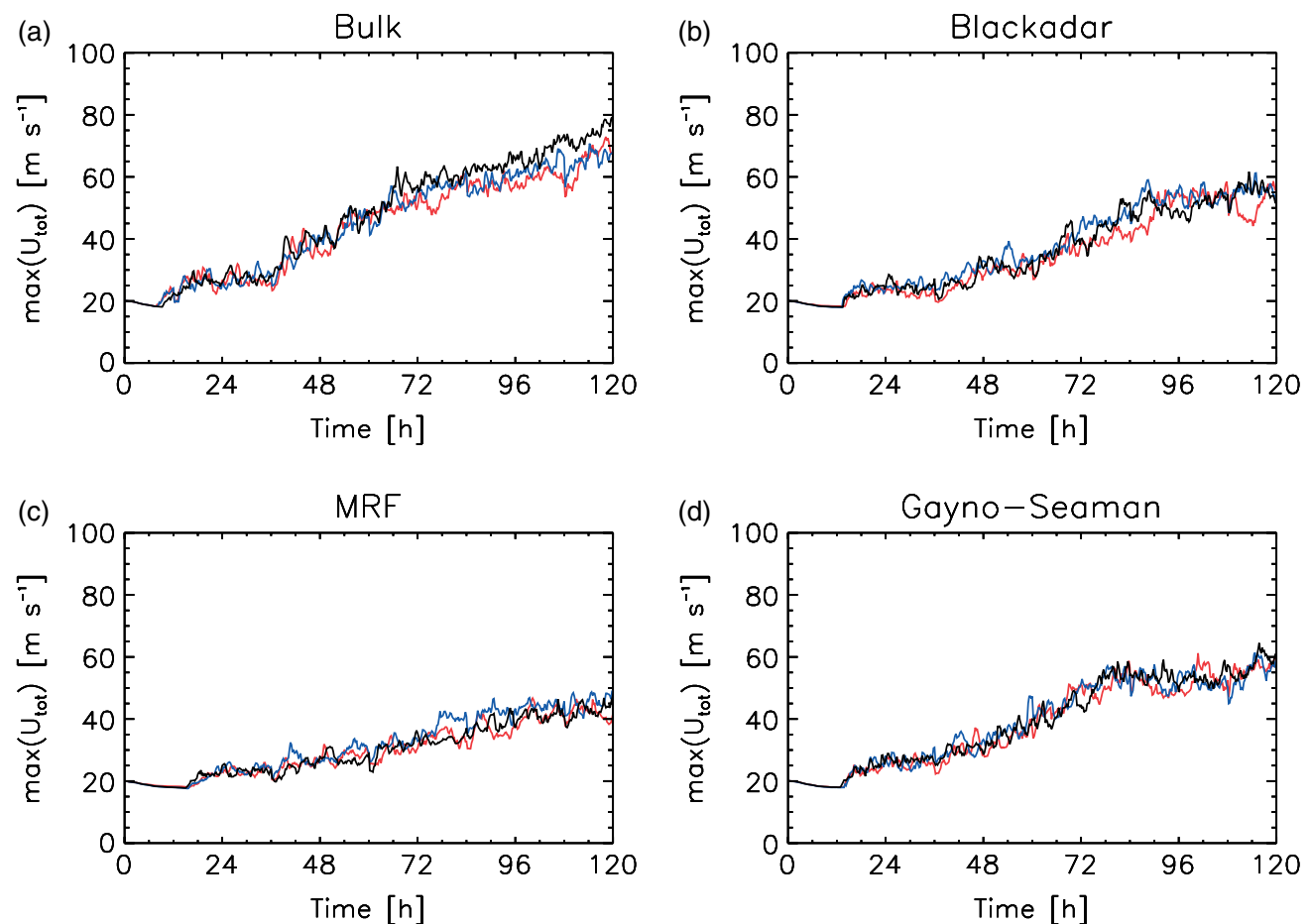

Figure 6. Time series of maximum wind speed in the calculations for a moving vortex with (a) the bulk scheme, (b) the Blackadar scheme, (c) the MRF scheme, and (d) the Gayno-Seaman scheme, for runs with a $-5 \mathrm{~m} \mathrm{~s}^{-1}$ uniform zonal wind field (black curve), runs with a $-5 \mathrm{~m} \mathrm{~s} \mathrm{~s}^{-1}$ uniform zonal wind field, with $5 \mathrm{~m} \mathrm{~s}^{-1}$ added to the zonal wind field for the calculation of the surface drag coefficient (red curve), and runs with a $-5 \mathrm{~m} \mathrm{~s}{ }^{-1}$ uniform zonal wind field, with $5 \mathrm{~m} \mathrm{~s}^{-1}$ subtracted from the meridional wind field for the calculation of the surface drag coefficient (blue curve).

The findings we described suggest that the precise behaviour of $C_{D}$ with surface wind speed may not be as critical as commonly thought. In essence, an approximate variation of $C_{D}$ with wind speed may be sufficient in coupled atmosphere-ocean models.

\section{Acknowledgements}

MTM acknowledges the support of grant no. N00014-031-0185 from the US Office of Naval Research, and the US National Science Foundation grant ATM-0715426. 

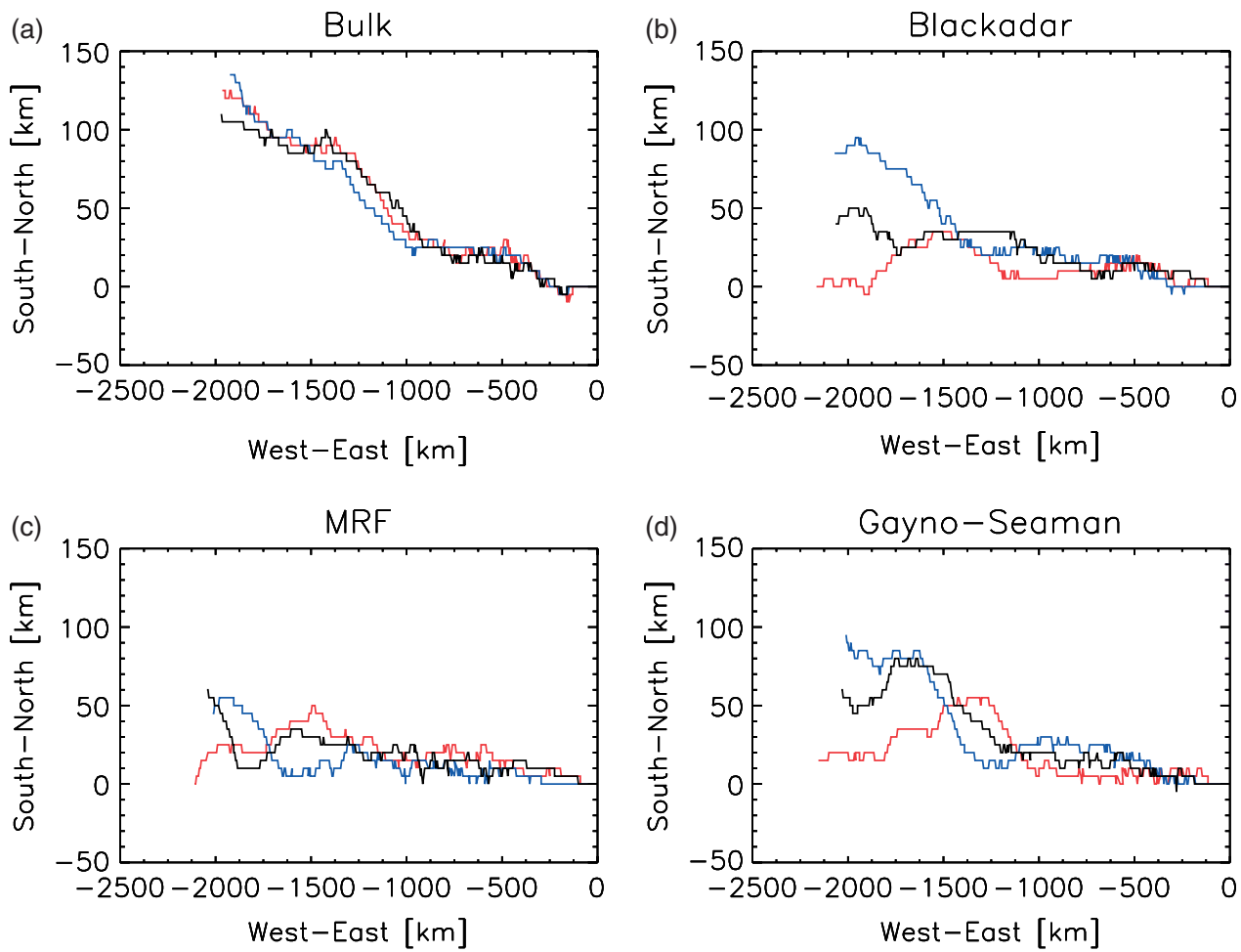

Figure 7. Track of the vortex centre (see text for definition) in the calculations for a moving vortex with (a) the bulk scheme, (b) the Blackadar scheme, (c) the MRF scheme, and (d) the Gayno-Seaman scheme, for runs with a $-5 \mathrm{~m} \mathrm{~s}^{-1}$ uniform zonal wind field (black curve), runs with a $-5 \mathrm{~m} \mathrm{~s}{ }^{-1}$ uniform zonal wind field, with $5 \mathrm{~m} \mathrm{~s}^{-1}$ added to the zonal wind field for the calculation of the surface drag coefficient (red curve), and runs with a $-5 \mathrm{~m} \mathrm{~s}{ }^{-1}$ uniform zonal wind field, with $5 \mathrm{~m} \mathrm{~s}^{-1}$ subtracted from the meridional wind field for the calculation of the surface drag coefficient (blue curve).

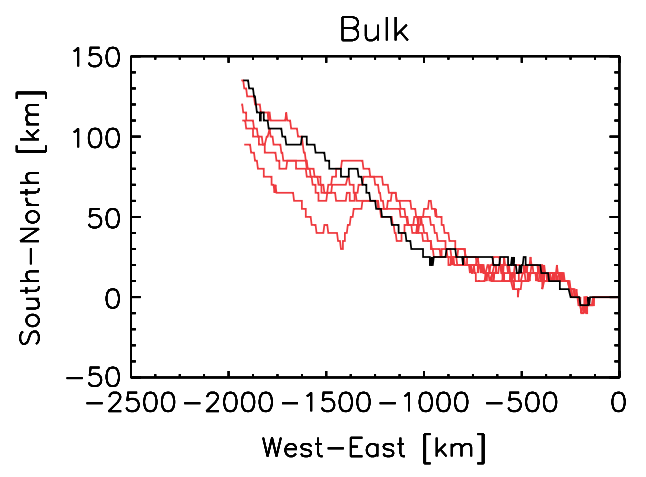

Figure 8. Track of the vortex centre (see text for definition) in the calculations with the bulk scheme for the control run as seen in Figure 7(a) (black curve), and four ensemble runs with random moisture perturbations and $\alpha=0$ (red curves).

\section{References}

Bell MM, Montgomery MT, Emanuel KA. 2012. Air-sea enthalpy and momentum exchange at major hurricane wind speeds observed during CBLAST. J. Atmos. Sci. DOI: 10.1175/JAS-D-11-0276.1.

Bister M, Emanuel KA. 1998. Dissipative heating and hurricane intensity. Meteorol. Atmos. Phys. 65: 233-240.

Black PG, D'Asoro EA, Drennan WM, French JR, Niller PP, Sanford TB, Terril EJ, Walsh EJ, Zhang JA. 2007. Air-sea exchange in hurricanes. Synthesis of observations from the Coupled Boundary-Layer Air-Sea Transfer experiment. Bull. Amer. Meteorol. Soc. 88: 357-374.

Braun SA, Tao W-K. 2000. Sensitivity of high-resolution simulations of hurricane Bob (1991) to planetary boundary-layer parameterizations. Mon. Weather Rev. 128: 3941-3961.

Chen SS, Price JF, Zhao W, Donelan MA, Walsh EJ. 2007. The CBLASTHurricane Program and the next-generation fully coupled atmosphere wave ocean models for hurricane research and prediction. Bull. Amer. Meteorol. Soc. 88: 311-317.
Donelan MA, Haus BK, Reul N, Plant WJ, Stiassnie M, Graber HC, Brown OB, Saltzman ES. 2004. On the limiting aerodynamic roughness of the ocean in very strong winds. Geophys. Res. Lett. 31: L18306, DOI: 10.1029/2004GL019460

Drennan WM, Zhang JA, French JR, McCormick C, Black PB. 2007. Turbulent fluxes in the hurricane boundary layer. Part II: Latent heat fluxes. J. Atmos. Sci. 64: 1103-1115.

Emanuel KA. 1986. An air-sea interaction theory for tropical cyclones. Part I: Steady-state maintenance. J. Atmos. Sci. 43: 585-604.

Emanuel KA. 1997. Some aspects of hurricane inner-core dynamics and energetics. J. Atmos. Sci. 54: 1014-1026.

Emanuel KA. 2012. Self-stratification of tropical cyclone outflow. Part II: Implications for storm intensification. J. Atmos. Sci. 69: 988-996.

French JR, Drennan WM, Zhang JA, Black PB. 2007. Turbulent fluxes in the hurricane boundary layer. Part II: Momentum fluxes. J. Atmos. Sci. 64: 1089-1102.

Grell GA, Dudhia J, Stauffer DR. 1995. 'A description of the fifth generation Penn State/NCAR mesoscale model (MM5)'. Tech. Note NCAR/TN-398+STR, NCAR: Boulder, Colorado.

Hill KA, Lackmann GL. 2009. Analysis of idealized tropical cyclone simulations using the Weather Research and Forecasting model: Sensitivity to turbulence parameterization and grid spacing. Mon. Weather Rev. 137: 745-765.

Jordan CL. 1958. Mean soundings for the West Indies area. J. Meteorol. 15: 91-97.

Kepert JD. 2006a. Observed boundary-layer wind structure and balance in the hurricane core. Part I. Hurricane Georges. J. Atmos. Sci. 63: 2169-2193.

Kepert JD. 2006b. Observed boundary-layer wind structure and balance in the hurricane core. Part II. Hurricane Mitch. J. Atmos. Sci. 63 : $2194-2211$.

Kepert JD, Wang Y. 2001. The dynamics of boundary-layer jets within the tropical cyclone core. Part II: Nonlinear enhancement. J. Atmos. Sci. 58: 2485-2501.

Large WG, Pond S. 1981. Open ocean momentum flux measurements in moderate to strong winds J. Phys. Oceanogr. 11: 324-336.

Montgomery MT, Smith RK, Nguyen SV. 2010. Sensitivity of tropical cyclone models to the surface exchange coefficients. Q. J. R. Meteorol. Soc. 136: 1945-1753. 
Moon IJ, Ginis I, Hara T. 2004. Effect of surface waves on air-sea momentum exchange. Part II: Behaviour of drag coefficient under tropical cyclones. J. Atmos. Sci. 61: 2334-2348.

Moon IJ, Ginis I, Hara T, Thomas B. 2007. A physics-based parameterization of air-sea momentum flux at high wind speeds and its impact on hurricane intensity predictions. Mon. Weather Rev. 135: 2869-2878.

Nguyen SV, Smith RK, Montgomery MT. 2008. Tropical-cyclone intensification and predictability in three dimensions. Q. J. R Meteorol. Soc. 134: 563-582.

Nolan DS, Zhang JA, Stern DP. 2009a. Evaluation of planetary boundary layer parameterizations in tropical cyclones by comparison of in-situ observations and high-resolution simulations of hurricane Isabel (2003). Part I: Initialization, maximum winds, and the outer core boundary layer. Mon. Weather Rev. 137: 3651-3674.

Nolan DS, Zhang JA, Stern DP. 2009b. Evaluation of planetary boundary layer parameterizations in tropical cyclones by comparison of insitu observations and high-resolution simulations of hurricane Isabe (2003). Part II: Inner core boundary layer and eyewall structure. Mon. Weather Rev. 137: 3675-3698.

Powell MD, Vickery PJ, Reinhold TA. 2003. Reduced drag coefficient for high wind speeds in tropical cyclones. Nature 422: 279-284.

Sanger NT. 2011. 'An observational study of tropical cyclone spin-up in supertyphoon Jangmi and hurricane Georges'. PhD dissertation, Naval Postgraduate school, Monterey, California. Available at: http://met.nps.edu/ mtmontgo/publications.html
Smith RK. 2006. Accurate determination of a balanced axisymmetric vortex. Tellus 58A: 98-103.

Smith RK, Montgomery MT. 2010. Hurricane boundary-layer theory. Q. J. R. Meteorol. Soc. 136: 1665-1770.

Smith RK, Thomsen GL. 2010. Dependence of tropical-cyclone intensification on the boundary-layer representation in a numerical model. Q. J. R. Meteorol. Soc. 136: 1671-1785.

Smith RK, Vogl S. 2008. A simple model of the hurricane boundary layer revisited. Q. J. R. Meteorol. Soc. 134: 337-351.

Smith RK, Montgomery MT, Nguyen SV. 2009. Tropical cyclone spin-up revisited. Q. J. R. Meteorol. Soc. 135: 1321-1335.

Smith RK, Montgomery MT, Thomsen GL. 2012. Sensitivity of tropical cyclone models to the surface drag coefficient in different boundarylayer schemes. Q. J. R. Meteorol. Soc. DOI:10.1002/qj.2057.

Thomsen GL, Smith RK, Montgomery MT. 2012. Tropical Cyclone report Nr.3. http://www.meteo.physik.uni-muenchen.de/ $\sim$ roger/Publications/M6.pdf

Zhang D-L, Liu Y, Yau MK. 2001. A multi-scale numerical study of hurricane Andrew (1992). Part IV: Unbalanced flows. Mon. Weather Rev. 129: 92-107.

Zhang JA, Black PB, French JR, Drennan WM. 2008. First direct measurements of enthalpy fluxes in the hurricane boundary layer: The CBLAST result. Geophys. Res. Lett. 35: L14813, DOI: 10.1029/2008GL034374

Zhang JA, Marks FD, Montgomery MT, Lorsolo S. 2011. An estimation of turbulent characteristics in the low-level region of intense hurricanes Allen (1980) and Hugo (1989). Mon. Weather Rev. 139: 1447-1462. 